\title{
An Introduction to Aquatic Toxicology [Introducción a la Toxicología acuática]
}

Nikinmaa, M. (2014). An Introduction to Aquatic Toxicology [Introducción a la Toxicología acuática] (240pp., ISBN 9780-12-411574-3). Ámsterdam: Academic Press.

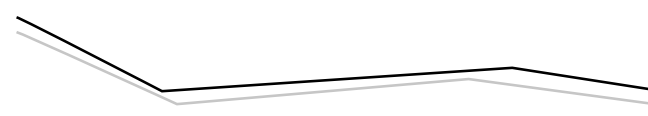

Roberto Rico Martínez*凶

Rico Martínez, R. (2017). An Introduction to Aquatic Toxicology [Introducción a la Toxicología acuática]. Reseña [Reseña de libro]. Investigación y Ciencia de la Universidad Autónoma de Aguascalientes, 25(72),131-132.

El Dr. Mikko Nikinmaa es profesor de Zoología del Departamento de Biología de la Universidad de Turku, Finlandia. Es, asimismo, editor en jefe de la revista Aquatic Toxicology. Escribió el libro An introduction to Aquatic Toxicology [Introducción a la Toxicología acuática, en español], con número de ISBN 978-0-12$411574-3$.

El libro cuenta con 240 páginas y no tiene desperdicio alguno. Contiene 18 capítulos que llevan al lector de la mano en los temas más recientes y relevantes en el área de la Toxicología acuática. En el primer capítulo hace una breve reseña de lo que es esta área, pasando por capítulos claves en los que explica las causas de la contaminación acuática, los principios de la purificación del agua, las fuentes y transporte de químicos en el agua, para pasar a los diseños experimentales y a la distribución de los químicos en organismos acuáticos, para finalizar con el modelaje de toxicidad.

En los primeros cinco capítulos abunda en el concepto de Toxicología acuática, las causas de la contaminación del agua, los principios de purificación de la misma, las fuentes de transporte de químicos en el ambiente acuático, y los más importantes diseños experimentales y organismos en Toxicología acuática. Con estos capítulos sienta las bases para que los lectores que inician en esta ciencia puedan entender los principios generales de esta disciplina.

\footnotetext{
* Departamento de Química, Centro de Ciencias Básicas, Universidad Autónoma de Aguascalientes. Av. Universidad 940, Ciudad Universitaria, C. P. 20131, Aguascalientes, Aguascalientes, México. Correo electrónico: rrico@correo.vaa.mx

$凶 \quad$ Autor para correspondencia
}

De los capítulos seis al ocho, el autor explica cuáles son los factores que afectan la biodisponibilidad de las sustancias químicas, cómo ocurre la entrada de las sustancias químicas a los organismos acuáticos, y cómo se lleva a cabo la distribución de las sustancias químicas en los organismos acuáticos. En estos capítulos utiliza una serie de esquemas, figuras y

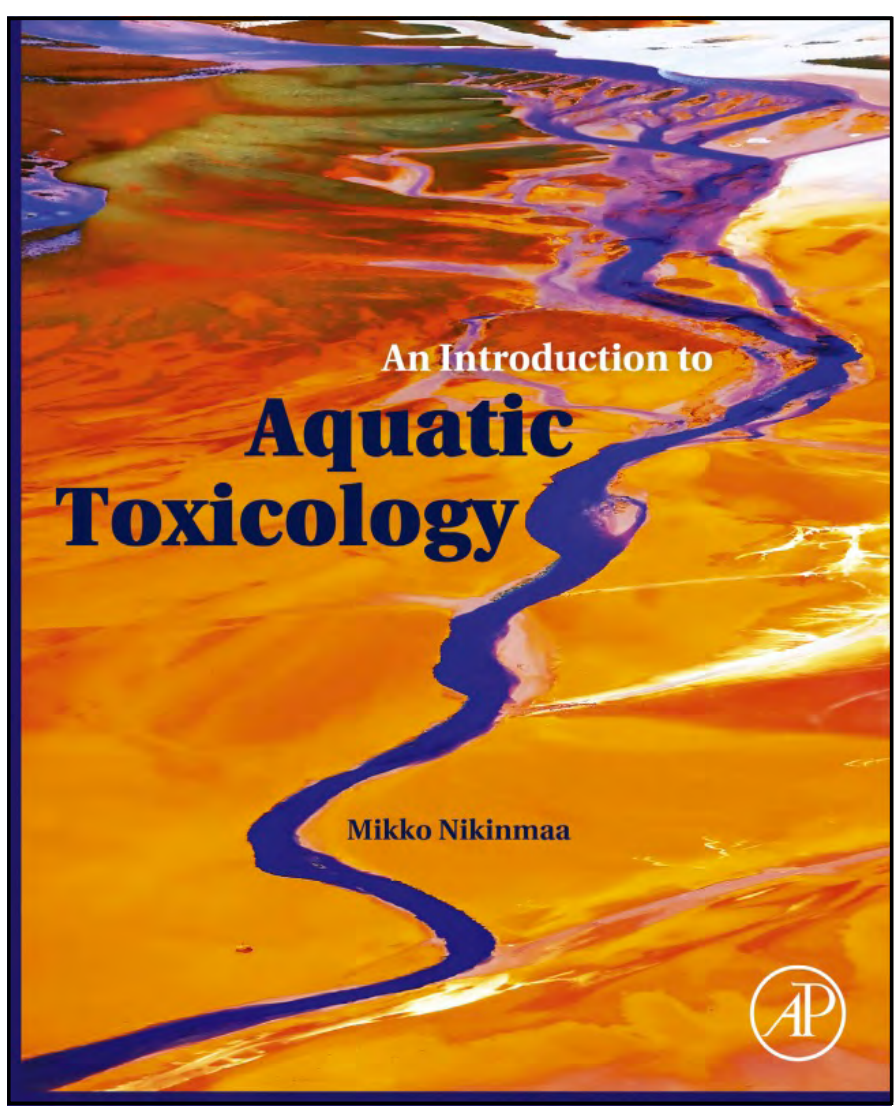

Portada del libro An Introduction to Aquatic Toxicology [Introducción a la Toxicología acuática]. 
IחVESTIGAGIÓn Y GIERCIA DE LA UNIVERSIDAD AUTÓกOMA DE RGUASCALIERTES cuadros de gran utilidad que facilitan el entendimiento de procesos clave.

Del capítulo nueve al quince es donde habla de los procesos en el interior de los organismos y su interacción con el ambiente, la parte medular del libro. Se tratan temas como la destoxificación y excreción de compuestos por los organismos y los efectos en estos. El capítulo doce está dedicado a los bioindicadores y biomarcadores, un tema particularmente relevante para los toxicólogos acuáticos. El sinergismo, antagonismo y demás interacciones entre sustancias químicas son cubiertas en el capítulo trece. Para el autor de la presente reseña fue particularmente agradable la lectura del capítulo catorce, dedicado a las pruebas de toxicidad aguda y crónica. En el capítulo quince se tratan las interacciones entre los factores ambientales naturales y la toxicidad.

Los efectos de las sustancias químicas en poblaciones acuáticas, así como en las comunidades acuáticas y ecosistemas, se tratan en los capítulos dieciséis y diecisiete. De nuevo una serie de figuras, esquemas y cuadros, explican en forma sencilla y concisa estas relaciones.

Finalmente, el capítulo dieciocho se titula: "Modelando la toxicidad". Es uno de los capítulos más interesantes y relevantes del libro. En forma sencilla explica conceptos difíciles como las relaciones entre la estructura cuantitativa y su relación con la actividad (QSAR's, por sus siglas en inglés: quantitative structure activity relationship), que se basa en que "la actividad de la molécula se basa en su estructura". También explica las bases de los modelos tóxico-cinéticos, en los que las propiedades del agua afectan la toxicidad de metales. Aquí explica las bases de la estimación del riesgo ambiental en forma amena y con profundidad.

Al concluir la lectura del libro no me resta más que otorgarle un completo endorso como un libro de texto para un curso de toxicología acuática en donde el autor demuestra por qué es el editor en jefe de una de las revistas más emblemáticas en el área: Aquatic Toxicology. 